E. Grün, Max-Planck-Institut für Kernphysik, Heidelberg, FRG and H.A. Zook, NASA Johnson Space Center, Houston, USA

Recent observations of zodiacal light have established a reliable and consistent picture of the spatial distribution of interplanetary dust in the ecliptic plane. The spatial density $n_{r}$ varies with heliocentric distance $r$ according to a power law $n_{r} \propto r^{-\nu}$. From Helios observations an exponent $\nu=1.3$ is derived for the distance interval from $0.08 \mathrm{~A}$.U. to 1 A.U. (Link et al. 1976). Outside the earth's orbit the Pioneer 10 and 11 results suggest a higher exponent $\nu=1.5$ for the distance interval from 1 A.U. to 3.3. A.U. (Hanner et al., 1976). Giese and Grün (1976) showed that the results from zodiacal light observations are compatible with the micrometeoroid fluxes derived from in situ measurements and lunar crater statistics. They found that micrometeoroids in the size range from $10 \mu \mathrm{m}$ to $100 \mu \mathrm{m}$ radii (corresponding roughly to $10^{-8} \mathrm{~g}$ to $10^{-5} \mathrm{~g}$ ) contribute most to the zodiacal light brightness.

The orbital distribution of large interplanetary particles $\left(10^{-6} \mathrm{~g}\right.$ $\left.<\mathrm{m}<10^{-3} \mathrm{~g}\right)$ is known from meteor observations. Sekanina and Southworth (1975) reported average orbital elements of these particles: $\bar{a} \sim 1.25$ A.U., $\overline{\mathrm{e}} \sim 0.4$ and $\bar{\gamma}_{\mathrm{i}} \sim 20^{\circ}$. Orbital information on micrometeoroids $\left(\mathrm{m}<10^{-8} \mathrm{~g}\right)$ is obtained from in situ detectors on board the Pioneer 8 and 9 and Helios 1 spaceprobes and the HEOS-2 satellite. Characteristics of the different micrometeoroid experiments are given in Table 1. There is almost no time overlap in the data taking intervals of the experiments. Therefore one has to assume that there are no time variations of the meteoroid flux on the time scale of 1 to 10 years if one compares the results from the different experiments. This assumption may be violated for the smallest of the observed particles ( $<10^{-13} \mathrm{~g}$ ) due to strong electromagnetic interaction of these particles with the interplanetary magnetic field (Morfill and Grün 1979).

\title{
OBSERVATIONAL EVIDENCE
}

Micrometeoroid detectors on board spaceprobes and satellites have observed a highly directional flux of interplanetary dust particles varying with particle sizes. The Pioneer 8 and 9 micrometeoroid experiments observed most of the smallest particles from the solar direction (Berg and Grün, 1973). They have been detected only by the front sensors 
Table 1: Characteristics of micrometeoroid experiments

\begin{tabular}{|c|c|c|c|c|c|}
\hline ïission & $\begin{array}{l}\text { data } \\
\text { period }\end{array}$ & orbit & $\begin{array}{l}\text { sensor } \\
\text { orientation }\end{array}$ & \begin{tabular}{|l|} 
field \\
of view
\end{tabular} & $\begin{array}{l}\text { sensitiv. } \\
\mathrm{v}=20 \mathrm{~km} / \mathrm{s}\end{array}$ \\
\hline Pioneer $8 * 1$ & $1968-72$ & $\begin{array}{l}\text { heliocentric } \\
0.98-1.08 \mathrm{AU}\end{array}$ & $\begin{array}{l}\text { scanning in } \\
\text { ecliptic plane }\end{array}$ & $160^{\circ} \mathrm{FFG}$ & $2 \times 10^{-13} \mathrm{~g}$ \\
\hline Pioneer $9 * 1$ & $1969-72$ & $\begin{array}{l}\text { heliocentric } \\
0.75-0.99 \mathrm{AU}\end{array}$ & $\begin{array}{l}\text { scanning in } \\
\text { ecliptic plane }\end{array}$ & $52^{\circ} \mathrm{TOF}$ & \\
\hline HEOS $-2 * 2$ & $1972-74$ & $\begin{array}{l}\text { geocentric } \\
7000 \mathrm{~km}- \\
245000 \mathrm{~km}\end{array}$ & $\begin{array}{l}\text { ecliptic north/south } \\
\text { earth apex/antapex }\end{array}$ & $120^{\circ}$ & $2 \times 10^{-16} \mathrm{~g}$ \\
\hline Helios $1 * 3$ & 1974 on & $\begin{array}{l}\text { heliocentric } \\
0.31-0.98 \mathrm{AU}\end{array}$ & $\begin{array}{l}\text { scanning in ecliptic } \\
\text { (ecliptic sensor), } \\
\text { scanning south } \\
\text { ecliptic hemisphere } \\
\text { (south sensor) }\end{array}$ & $\begin{array}{l}130^{\circ} \\
140^{\circ}\end{array}$ & $4 \times 10^{-14} \mathrm{~g}$ \\
\hline
\end{tabular}

$*^{1}$ : Berg and Richardson 1969

$*^{2}$ : Hoffman et a1. 1975

$*^{3}$ : Grün et al. 1979

(FFG events). These particles ( $\beta$-meteoroids) are on hyperbolic orbits leaving the solar system due to the action of radiation pressure (Zook and Berg, 1975). They have also been identified in the data of the micro-meteoroid detector on board Helios 1 (Grün et a1., 1979).

Another class of meteoroids, intermediate in mass between the $\beta-$ meteoroids described above and the larger "sporadic" meteoroids, had also been identified in the data from each of four separate experiments. These intermediate mass particles are observed to arrive from the heliocentric spacecraft apex direction in each case. As we shall show, these intermediate mass meteoroids appear to constitute a dynamical class of meteoroids that is separate from both the larger sporadic meteoroids spiraling in toward the Sun under $P-R$ drag and the smaller $\beta$-meteoroids on hyperbolic trajectories. We sha11, for convenience, give this class of intermediate mass meteoroids a name: " $\alpha$-meteoroids."

In the Pioneer 8 and 9 meteoroid data, these particles are represented by the largest FFG events (McDonne11, 1978) as well as particles which penetrated the front film and were additionally registered by the rear sensor (TOF events, Berg and Gerloff, 1971). The ecliptic sensor of the Helios 1 space probe similarly detected most of these intermediate mass meteoroids from the apex direction (Grün et al., 1979). Also the HEOS-2 experiment clearly observed these particles from the earth's apex (Hoffmann et al., 1975). Figure 1 shows the flux of dust particles detected in interplanetary space (outside 10 earth radii) from the 4 principal pointing directions. Below $10^{-11} \mathrm{~g}$ the flux from the apex direction rises steeply and is about an order of magnitude higher than from the outer direction between $10^{-11} \mathrm{~g}$ and $10^{-14} \mathrm{~g}$. This mass range is 


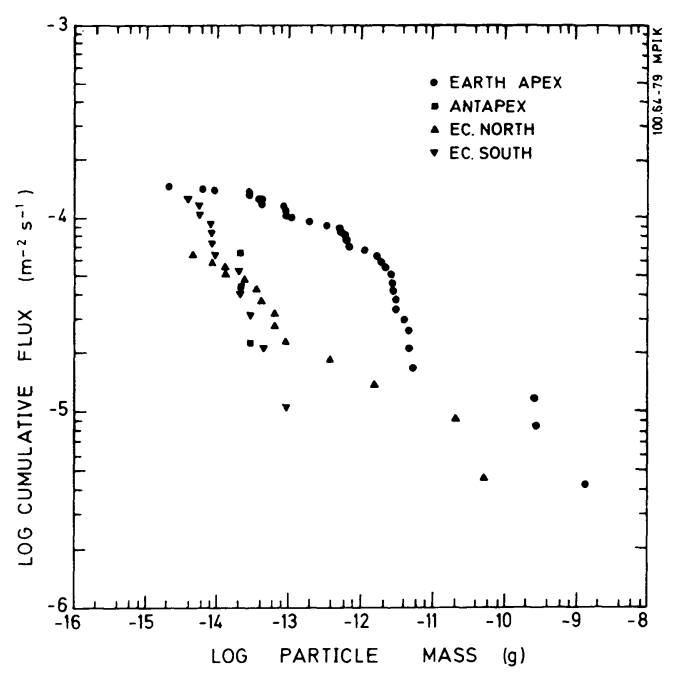

Figure 1: Cumulative interplanetary particle flux as observed by HEOS-2 from 4 principal pointing directions.

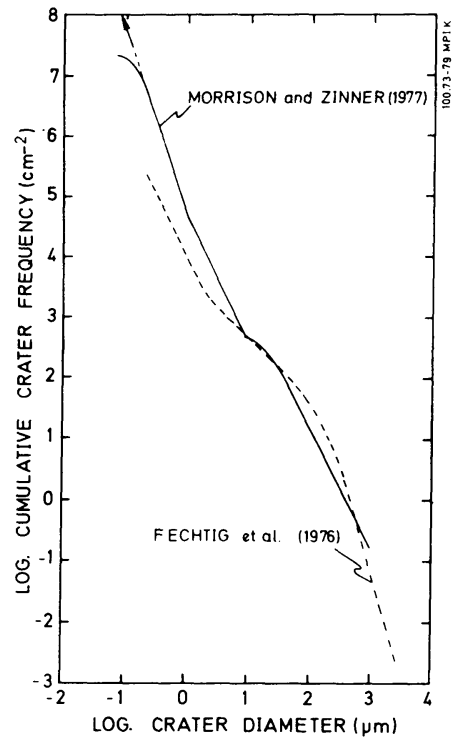

Figure 2: Crater size frequency on lunar rocks.

in agreement with the mass range of the Pioneer 8/9 TOF events reported by McDonnell (1978). The average impact speed of these meteoroids onto the HEOS-2 sensor is $10 \mathrm{~km} / \mathrm{s}$ with an upper limit of $23 \mathrm{~km} / \mathrm{s}$ (Hoffmann et a1., 1975). These observations indicate low angular momentum particles which are detected close to their aphelion. Such orbits will also explain the strong concentration of radiants towards the ecliptic plane.

The flux of the largest particles detected by the HEOS-2 sensor $\left(\mathrm{m}>10^{-1} \mathrm{~g}\right)$ show no pronounced directionality in the plane perpendicular to the heliocentric radius vector. This is similar to the apparent radiant distribution observed for the sporadic meteors (Sekanina and Southworth, 1975). In the ecliptic plane Helios 1 detected no strong directionality of the large particle flux. The radiant distribution of large micrometeoroids is significantly different from the distribution of $\alpha$-meteoroids. Therefore we conclude that the large particles (sporadic meteoroids) and $\alpha$-meteoroids belong to different populations.

These three groups of dynamically different micrometeoroids also should show up in the lunar microcrater statistics. Figure 2 presents the microcrater size-frequency reported by Fechtig et al. (1976) and Morrison and Zinner (1977). While the Morrison and Zinner data were obtained from a single sample (12054) the Fechtig et al. data are a compilation of data from different samples, which may explain the differences between the two distributions. The sporadic meteoroid population with masses $\mathrm{m}>10^{-11} \mathrm{~g}$ corresponds to craters with pit diameters $\mathrm{d}>10 \mu \mathrm{m}$ (Hörz et al., 1975). These large craters (10 $\mu \mathrm{m}<\mathrm{d}<1 \mathrm{~mm}$ ) link the sporadic meteors with the micrometeoroids accessible to in situ detectors. The bending over at smaller masses of sporadic meteoroids is explained by the fact that radiation pressure limits the input of small particles from comets into bound orbits (Dohnanyi, 1972). For pit 
diameters between about $1 \mu \mathrm{m}$ and $10 \mu \mathrm{m}$ (corresponding to $10^{-13} \mathrm{~g}$ and $10^{-11} \mathrm{~g}$ ) the $\alpha$-meteoroid population should be seen. In this crater size range the discrepancy in the lunar data is large, which may be partially explained by different shielding of the samples. Below $d \sim 1 \mu \mathrm{m}$ the $\beta-$ meteoroid population should dominate. The transition between the $\beta$ and $\alpha$ meteoroid populations is not yet clearly observed in the lunar impact pit data but a more detailed look into lunar impact pit populations should reveal this transition more clearly.

THE COLLISIONAL MODEL

Sporadic meteoroids are injected into interplanetary space by comets (Whipple, 1967 and Dohnanyi, 1972). They are dispersed by the action of Poynting-Robertson effect and collisions. Mutual collisions between micrometeoroids determine the life-time of dust particles with masses $\mathrm{m}>10^{-6} \mathrm{~g}$ (Whipple, 1968; Zook and Berg, 1975 and Dohnanyi, 1978). Most collisions appear to occur close to the sun, where the spatial density of dust particles is highest. The number of collisions $f(r)$ per radial distance element $\mathrm{dr}$ is given by

$$
f(r) d r=n_{r}{ }^{2} \bar{v} 4 \pi r^{2} d r
$$

The spatial density is taken $\mathrm{n}_{\mathrm{r}} \propto \mathrm{r}^{-1.3}$ for 0.05 A.U. $<\mathrm{r} \leq 1$ A.U., $\mathrm{n}_{\mathrm{r}}=$ const for $0.02 \mathrm{~A} . \mathrm{U} .<\mathrm{r} \leq 0.05 \mathrm{~A} . \mathrm{U}$. and $\mathrm{n}_{\mathrm{r}}=0$ inside $\mathrm{r}=0.02 \mathrm{~A} . \mathrm{U}$. The average collision speed $\bar{v}$ is proportional $\mathrm{r}-0.5$. Figure 3 shows the

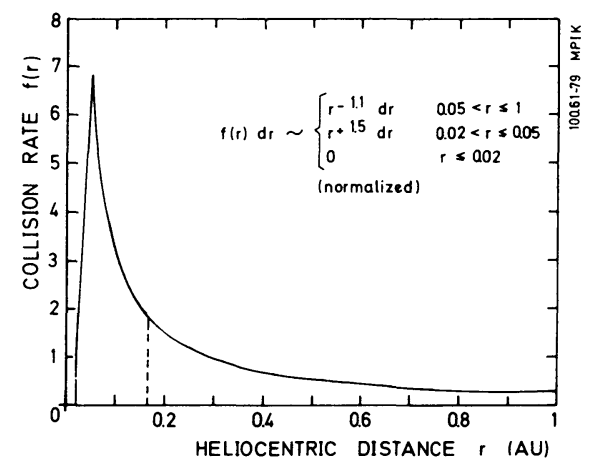

Figure 3: Number of meteoroid collisions per unit distance as function of heliocentric distance.

number of collisions as a function of heliocentric distance. Most of the collisions between particles of the zodiacal light cloud take place close to the Sun; half of them inside $0.16 \mathrm{~A} . \mathrm{U}$.

The very small collision fragments will experience sufficient radiation pressure compared to gravity to put them into hyperbolic orbits-the $\beta$-meteoroids. The term $\beta$ is often used to represent the ratio between the radiation pressure force and the gravitational force $\left(B=\mathrm{F}_{\mathrm{rad}} / \mathrm{F}_{\mathrm{grav}}\right)$ and here describes particles where this ratio is high. Zook and Berg (1975) and Dohnanyi (1976) demonstrated that collisions among the larger meteoroids provide a sufficient source for the observed $\beta$-meteoroids. Most of them are produced by collisions inside $0.2 \mathrm{~A} . \mathrm{U}$. These particles are small enough that even electromagnetic effects will strongly influence their trajectories (Morfill and Grün, 1979). 
Larger collisional fragments, with smaller $\beta$ values, will not be injected into hyperbolic orbits after release from their parent bodies but will have aphelia and eccentricities greatly increased over the parent obj€cts. This dynamical grouping is here called the $\alpha$-meteoroid group. If a parent meteoroid with an orbit eccentricity $e_{p}$ is hit by a small particle at the distance $r$ in A.U. from the Sun, then the fragments will reach the Earth's orbit if their radiation pressure constants are $\beta_{\min } \leq \beta<\beta_{\max }$ with $\beta_{\min }=\left(1-e_{\mathrm{p}}-\mathrm{r}-\mathrm{e}_{\mathrm{p}} \mathrm{r}\right) / 2 \geq 0$ and $\beta_{\max }=\left(1-\mathrm{e}_{\mathrm{p}}\right) / 2$. Particles with $\beta \geq \beta_{\max }$ are $\beta$-meteoroids which leave the solar system. Most of the particles with $\beta<\beta_{\max }$ have low angular momentum and will therefore be detected by a micrometeoroid detector at 1 A.U. from the apex direction ( $\alpha$-meteoroids).

The range of possible radiation pressure constants of $\alpha$-meteoroids decreases close to the Sun which corresponds also to a decreasing mass interval. Therefore the contribution to $\alpha$-meteoroids from collisions in the inner solar system is low. If we assume eccentricities of the parent bodies $e_{p}=0.5$ then the highest observation probability is for particles which are produced outside $0.3 \mathrm{~A} . \mathrm{U}$. From the fact that the maximum observed impact speed of $\alpha$-meteoroids onto the HEOS-2 sensor is $23 \mathrm{~km} / \mathrm{s}$ and that they have not been observed from the ecliptic north and south direction, a maximum distance from the Sun can be calculated at which most fragmentation takes place. If we take an average inclination $i \sim 20^{\circ}$ of the parent meteoroids then the fragments will stay within the experimentally observed limits if most of the collisions took place inside 0.8 A.U.

This interpretation of the $\alpha$-meteoroids is in agreement with the results of the Helios 1 experiment, which observed $\alpha$-meteoroids by the ecliptic sensor with semimajor axes $a \leq 0.5$ or eccentricities $\mathrm{e}<0.4$ and an average inclination of $i \sim 20^{\circ}$ (Grün et al. 1979). The densities of dust particles derived by Pailer and Grün (1980) from the Helios 1 data for different groups of particles support the interpretation given above. The big particles with low densities $\left(\rho<1 \mathrm{~g} / \mathrm{cm}^{3}\right)$ detected by the south sensor belong to the sporadic meteoroid population which originates directly from comets, whereas the $\alpha$-meteoroids have normal densities $\left(\rho>1 \mathrm{~g} / \mathrm{cm}^{3}\right)$ as expected for collisional fragments of the sporadic meteoroid population.

\section{REFERENCES}

Berg, O.E. and Gerloff, U.: 1971, Space Research XI, p. 225.

Berg, O.E. and Grün, E.: 1973, Space Research XIII, p. 1047.

Berg, O.E. and Richardson, E.F.: 1969, Rev. Sci. Instrum., 40, p. 1333.

Dohnanyi, J.S.: 1972, Icarus, 17, p. 1.

Dohnanyi, J.S.: 1976, Lecture Notes in Physics, 48, p. 170.

Dohnanyi, J.S.: 1978, in "Cosmic Dust", ed. J.A.M. McDonne11, p. 527.

Fechtig, H., Gentner, W., Hartung, J.B., Nage1, K., Neukum, G.,

Schneider, E. and Storzer, D.: 1976, NASA SP-370, p. 585.

Giese, R.H. and Grün, E.: 1976, Lecture Notes in Physics, 48, p. 135.

Grün, E., Pailer, N., Fechtig, H. and Kissel, J.: 1979, submitted to Planet. Space Sci. 
Hanner, M.S., Sparrow, J.G., Weinberg, J.L. and Beeson, D.E.: 1976, Lecture Notes in Physics, 48, p. 24.

Hoffmann, H.J., Fechtig, H., Grün, E. and Kissel, J.: 1975, Planet. Space Sci., 23, p. 985.

Horz, F., Brownlee, D.E., Fechtig, H., Hartung, J.B., Morrison, D.A, Neukum, G., Schneider E., Vedder, J.E. and Gault, D.E.: 1975, Planet. Space Sci., 23, p. 151.

Link, H., Leinert, C., Pitz, E. and Salm, N.: 1976, Lecture Notes in Physics, 48, p. 19.

McDonne11, J.A.M.: 1978, in "Cosmic Dust", ed. J.A.M. McDonne11, p. 337. Morfill, G.E., and Grün, E.: 1979, Planet. Space Sci., to be published. Morrison, D.A. and Zinner E.: 1977, Proc. 8th. Lunar Sci. Conf., p. 841. Pailer, N. and Grün, E.: 1980, this volume.

Sekanina, Z. and Southworth, R.B.: 1975, NASA CR-2615. Whipple, F.L.: 1967, NASA SP-150, p. 409.

Zook, H.A. and Berg, O.E.: 1975, Planet. Space Sci., 23, p. 183.

\section{DISCUSSION}

Hughes: Have you evaluated other sources for your $\beta$-meteoroids? For example, comets as a primary source, and spin-induced fragmentation of particles.

Misconi: I also think that rotational bursting of the dust, creating submicron grains from larger parent particles, is a likely source. Grin: So far, Helios has not observed any strong grouping of impacts correlated with a known comet or meteor stream. Therefore this does not support the Explorer 46 findings. On the other side, the mass loss rate determined from the well-established radial and size dependence of micrometeoroids can account for most of the observed flux of $\beta$-meteoroids if you consider only collisions among the larger particles.

Hughes and Elford: It must be remembered also that radar meteor observations have a builtin bias toward high-velocity, large-mass objects and conservatively record $<10 \%$ of the mass striking the Earth in their sensitivity range. 